\title{
RELACIÓN DEL PRESUPUESTO POR RESULTADOS (PpR) EN LA EJECUCIÓN DE LOS SERVICIOS ODONTOLÓGICOS EN LA ESTRATEGIA SANITARIA DE SALUD BUCAL DIRESA DE TACNA 2012
}

\section{LIST OF BUDGET RESULTS (RBB) IMPLEMENTATION OF DENTAL HEALTH SERVICES IN ORAL HEALTH STRATEGY DIRESA TACNA 2012}

\author{
Sánchez-Macchiavello Alonso. ${ }^{1}$ \\ ${ }^{1}$ Magíster en Odontoestomatología, Docente de la Escuela Profesional de Estomatología de la Universidad Alas Peruanas, \\ Cirujano dentista en el Centro de Salud La Esperanza en la cuidad de Tacna.Perú. \\ *alonso.fsm@hotmail.com
}

\begin{abstract}
Resumen
OBEJETIVO El presente estudio tuvo como objetivo principal, estudiar la relación que existe entre el gasto público y la cantidad de atenciones y atendidos, en las diferentes redes y micro redes de la DIRESA TACNA. Se estudiaron todas las redes de salud de Tacna en el período 2012 obteniéndose además el consolidado de atenciones y atendidos del registro de estadística HIS del Ministerio de Salud y el gasto de la página web del ministerio de Economía del Perú. La técnica fue observación documental, el análisis estadístico descriptivo que se empleó fue de frecuencias y porcentajes. Los resultados encontrados, nos mostraron que hay una buena ejecución en tratamientos preventivos y recuperativos, y cero actividad en tratamientos especializados, motivo por el cual Tacna, quedó en último lugar de ejecución de estas labores en comparación con otras regiones. El presente estudio demostró mediante la observación de los resultados sanitarios del reporte HIS, que en las Redes de Salud con poca población asignada, el gasto no corresponde a la cantidad de atenciones a diferencia de las otras redes donde el volumen de compra y gasto de recursos es concordante con la ejecución de metas de actividades. La hipótesis se comprobó, mediante la prueba de regresión lineal y el R cuadrado de Pearson.
\end{abstract}

Palabras clave: Presupuesto, salud bucal, estrategia sanitaria.

\section{Abstract}

AIM:The aim of this study was to determinate the relationship between public spending and the amount of attention and oral health services done, at different micro networks from DIRESA TACNA. Every health micro networks of Tacna were studied in the period 2012, obtaining also the consolidated number of attentions and attended from the register of statistical HIS office from the website of the Economy Ministry of Peru. The technique used was documental observation; the descriptive statistics used were of frequencies and percentages. The result found, we showed that there is a satisfactory, preventive and recuperative preformance, and zero activity in specialized treatments. Tacna, showed the last execution of these tasks compared with other regions in Peru. The present study showed that the expenditure in the Health Network with small populations does not correspond to the amount of attention; unlike the Networks where volume purchase and the expenditure of resources is consistent with the goals of implemented activities. The hypothesis was tested and probed by linear regression test and Pearson R square.

Key words: Budget, oral health, health strategy.

\section{INTRODUCCIÓN}

La constitución política del Perú, en el artículo 1 dice explícitamente: " "La defensa de la persona humana y el respeto de su dignidad son el fin supremo de la sociedad y del Estado". En el artículo 2 reconoce el derecho de la persona a la vida y al concebido como sujeto de derecho en todo en cuanto le favorece. En el artículo 4 indica explícitamente 
"La comunidad y el Estado protegen especialmente al niño, al adolescente, a la madre y al anciano en situación de abandono. También protegen a la familia y promueven el matrimonio. Reconocen a estos últimos como institutos naturales y fundamentales de la sociedad..." en su artículo 7 declara que la salud es un derecho de todos los peruanos, indicando que todos tiene derecho a la protección de su salud, así mismo en el artículo 9 se menciona explícitamente "El Estado determina la política nacional de salud. El Poder Ejecutivo norma y supervisa su aplicación. Es responsable de diseñarla y conducirla en forma plural y descentralizadora para facilitar a todos el acceso equitativo a los servicios de salud". Finalmente el artículo 11 de la constitución garantiza el libre acceso a prestaciones de salud. En conclusión en el Perú la salud es un derecho, sobre todo de los más pobres, quintiles 1 y 2 de pobreza. En el marco de la Ley General de Salud Nro 26842; en atención al artículo 3 del Decreto Supremo Nro 016-2005-SA, Reglamento de la Ley de trabajo del Cirujano Dentista que a la letra dice: "El Cirujano Dentista participa en la aplicación de las políticas de salud, desarrollando y ejecutando las acciones de prevención, recuperación y rehabilitación de la salud de las personas, la familia y la comunidad, brindándole además educación, orientación y asesoría en salud estomatológica dentro del ámbito de la salud integral", en concordancia con el artículo 12 del mismo Decreto Supremo que respecto al trabajo asistencial dice "El trabajo asistencial es el dedicado a la consulta y atención estomatológica integral al consultante, que comprende la promoción, prevención, diagnóstico, recuperación y rehabilitación de la salud del sistema estomatognático, así como su participación en el equipo multidisciplinario de salud que brinda atención integral estomatológica al paciente". ${ }^{2}$ La R.M.689-2006/MINSA, establece esta herramienta para "la estandarización, alineamiento e intercambio de información de la producción de servicios de salud y es la base para determinación de la cartera de servicios de cada establecimiento prestador público y privado, así como es el insumo más importante para la determinación de Unidades Relativas de Valor en el marco de la política tarifaria del sector". 4

El Ministerio de Salud brinda tres tipos de prestaciones odontológicos; Primero, las atenciones preventivas, que consisten en diferentes aplicaciones en los exámenes odontológicos, la fisioterapia, la asesoría nutricional, destartraje, profilaxis dental y aplicación de flúor; Segundo, las atenciones Recuperativas son las exodoncias, las obturaciones con resina; y Tercero, las atenciones especializadas, las cuales se dan en los centros de Salud de mayor nivel de complejidad y las realizan los endodoncistas, periodoncistas y odontopediatras.

Adicionalmente, sabemos que en nuestro país las enfermedades bucales constituyen un grave problema de Salud Pública debido a la alta prevalencia de las mismas, donde la caries dental constituye una de las primeras causas de morbilidad general, enfermedad que aqueja a más del $90 \%$ de la población nacional y a más del $95 \%$ de la Regional.

La Ley de presupuesto del Sector Público Nro 29465 para el año 2010, en su cuadragésima disposición final dispone la creación del Aplicativo para la Gestión y Seguimiento de Insumos y Productos en Presupuesto por Resultados (SIP PpR), a cargo de la Dirección General del Presupuesto Público del Ministerio de Economía y Finanzas, que tiene por objeto registrar, verificar y monitorear el uso de los insumos necesarios para la prestación de los productos o bienes y servicios públicos. ${ }^{2}$ En el marco del mandato legal, La Dirección General del Presupuesto Público (DGPP), continúa desarrollando diversos procedimientos operativos para implementar el Presupuesto por Resultados en el Perú. Uno de los cambios más importantes está referido a la programación y formulación presupuestaria, como fases iniciales del proceso, en las que se identifican las intervenciones a financiar y las metas a alcanzar. Dotar a estas fases del enfoque de resultados requiere, naturalmente, especificar los procedimientos a seguir, en tal sentido, en el marco de la aplicación progresiva del Presupuesto por Resultados en el Perú, se continúa con los procesos de diseño de intervenciones, orientados a la generación de resultados, que puedan propiciar la efectividad en el desarrollo del país, considerando que el $\mathrm{PpR}$ asocia el gasto público con la entrega de productos y la obtención de resultados, es fundamental que la programación y asignación de los recursos se oriente en ese sentido, por lo que la DGPP, puso a disposición de los equipos de gestión de las unidades ejecutoras ( Redes de Salud) de los pliegos regionales, la segunda versión del instrumento metodológico, para la Programación y Formulación de Metas Estratégicas, que les permitirá obtener un presupuesto real y personalizado por cada uno de los puntos de atención, en la lógica de resultados.

El presente trabajo de investigación tuvo por finalidad evaluar la correlación entre el presupuesto asignado estratégicamente y la ejecución de atenciones dentales en la Red de Salud Tacna, el presente trabajo se presenta de acuerdo al reglamento de la escuela de post grado, un primer capítulo único donde se exponen los resultados de la investigación, luego las conclusiones, sugerencias y propuesta, en la parte final se presentan los anexos, que lleva entre ellos el proyecto de investigación que dio origen a este informe.

Dado que: El presupuesto por resultados es una metodología nueva en el sector público, Es probable que: La cantidad de presupuesto no guarde una relación estadísticamente significativa con la cantidad de tratamientos ejecutados en las distintas redes de la región Tacna. 


\section{MATERIALES Y MÉTODOS}

El departamento de Tacna se encuentra ubicado en el extremo sur del país, en un apacible y soleado valle a 552 msnm. Por el norte limita con el Moquegua, por el sur con Chile, por el este con Puno y Bolivia y por el Oeste con el Océano Pacífico. Su clima es templado, con una temperatura máxima en verano de 28 grados centígrados. Tiene cuatro provincias estas son: Jorge Basadre, Tarata, Candarave y Tacna, contando con 26 distritos en toda la ciudad.

El presente trabajo hace referencia a la ejecución presupuestal del Año fiscal 2012. Visión Temporal. Transversal. Corte temporal. Retrospectivo Las Unidades de estudio fueron: Las 09 Micro Redes de Salud de la región de Tacna, que son:

1) Micro Red Alto Perú.

2) Micro Red Candarave.

3) Micro Red Cono Norte.

4) Micro Red Litoral.

5) Micro Red Metropolitana.

6) Micro Red Tarata.

7) Micro Red Cono Sur.

8) Micro Red Frontera.

9) Micro Red Jorge Basadre

Para la toma de datos se hizo la coordinación con los trabajadores de estadística con la finalidad de solicitar la cantidad de atenciones y atendidos mediante el reporte 40 de las fichas HIS. MIS. Coordinación con los trabajadores de la oficina de planificación y presupuesto, a fin de obtener los datos de Presupuesto por Resultados de la Región Tacna, la misma que obra en la página web del ministerio de Economía. Se realizó la validación del instrumento mediante una prueba piloto, donde se evaluará la eficacia de la ficha de toma de datos. Para manejar los resultados

A nivel de sistematización: Se generó una base de datos en

Excel y luego se analizó las frecuencias y porcentajes.

A nivel de análisis de los datos: Se ingresó a la consulta amigable del ministerio de economía y finanzas y se obtuvo la información de atendidos y atenciones del reporte 40 de la oficina de Estadística del Ministerio de Salud. http://ofi.mef.gob.pe/transparencia/Navegador/default.aspx

A nivel de conclusiones: Se redactarán ejecutando los objetivos y contestando las interrogantes básicas.

\section{RESULTADOS}

Podemos apreciar que la mayor parte de atendidos, son los que acudieron por motivos preventivos $160.836 \mathrm{de}$ 222.618 pacientes, mientras que en los recuperativos acudieron 61.782 de 222.618. las especializadas dieron un número de 0 atentidos.(cuadro 1 y gráfico 1)

Podemos ver que las redes Cono norte , Metropolitana, Cono sur son las que aglomeran la mayor cantidad de atendidos y atenciones preventivas ; al comparar 9 microredes la

\begin{tabular}{lll}
\hline & $\mathrm{N}$ & $\%$ \\
\hline Preventivo & $\mathbf{1 6 0 , 8 3 6}$ & $\mathbf{7 2 . 2 5}$ \\
Recuperativo & 61,782 & 27.75 \\
Especializado & 0 & 0.00 \\
TOTAL & 222,618 & 100.00 \\
\hline
\end{tabular}

Tabla 1. Frecuencia de las prestaciones en cuanto a pacientes atendidos en Tacna 2012

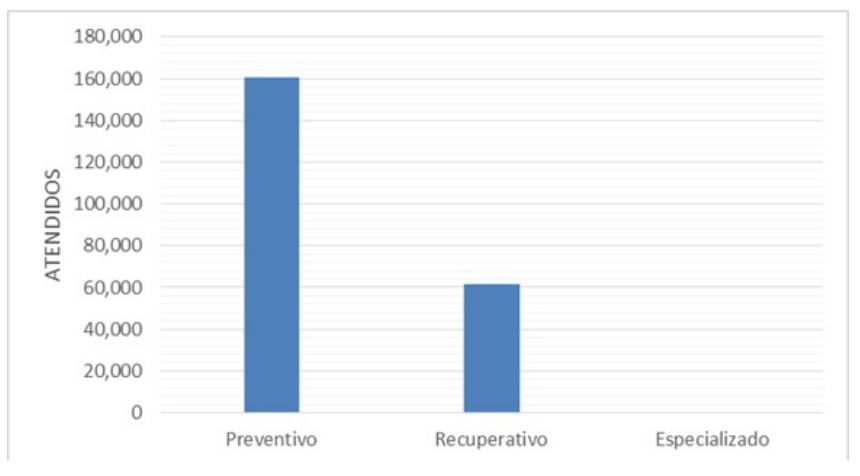

Grf. 1. Frecuencia de las prestaciones en cuanto a pacientes atendidos, pacientes de Tacna

aglomeracion de las 3 microredes excede el $50 \%$ del total, cumpliendo con la labor del primer nivel de atención, es decir trabajando con los pacientes sanos. La mayor concentracion entre atendidos y atenciones es de 2.1 de la microred Tarata..(tabla 2 y gráfico 2).

Podemos ver que las redes Cono norte , Metropolitana, Cono Sur son las que aglomeran la mayor cantidad de atendidos y atenciones recuperativas, cumpliendo con la labor asistencial a los pacientes enfermos, lo que generá que las

\begin{tabular}{llll}
\hline MICRO REDES & Atendidos & Atenciones & Concentración \\
\hline Alto Perú & 687 & 1,151 & 1.7 \\
Candarave & 4,527 & 6,915 & 1.5 \\
Cono norte & 28,698 & 44,451 & 1.5 \\
Litoral & 4,683 & 7,636 & 1.6 \\
Metropolitana & 24,434 & 44,975 & 1.8 \\
Tarata & 3,880 & 8,033 & 2.1 \\
Cono sur & 23,613 & 31,902 & 1.4 \\
Frontera & 4,217 & 7,965 & 1.9 \\
Jorge Basadre & 4,187 & 7,808 & 1.9 \\
Total & 98,926 & 160,836 & 1.6 \\
\hline
\end{tabular}

Tabla 2. Frecuencia de las prestaciones preventivas en cuanto a atendidos y atenciones 


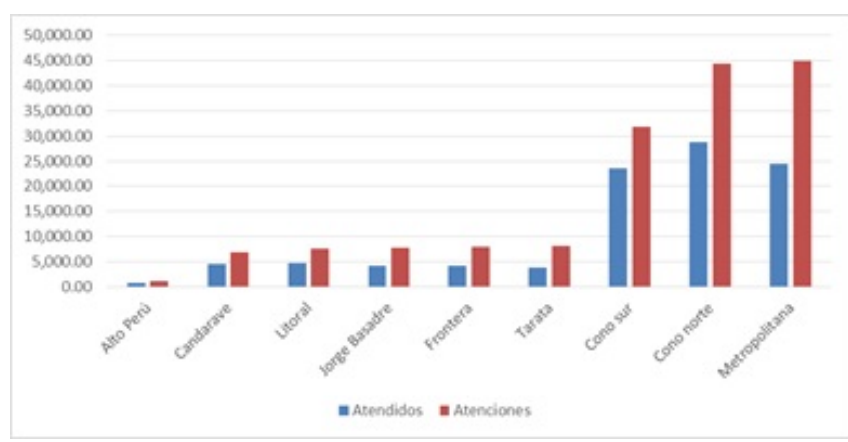

Grf. 2. Frecuencia de las prestaciones preventivas en cuanto a atendidos y atenciones.

\begin{tabular}{llll}
\hline $\begin{array}{l}\text { MICRO } \\
\text { REDES }\end{array}$ & Atendidos & Atenciones & Concentración \\
\hline Alto Perú & 295 & 400 & 1.4 \\
Candarave & 1741 & 2366 & 1.4 \\
Cono norte & 14229 & 18898 & 1.3 \\
Litoral & 1408 & 1651 & 1.2 \\
Metropolitana & 11762 & 15873 & 1.3 \\
Tarata & 1179 & 1704 & 1.4 \\
Cono sur & 11677 & 14532 & 1.2 \\
Frontera & 3053 & 3442 & 1.1 \\
Jorge & 2166 & 2916 & 1.3 \\
Basadre & 47510 & 61782 & 1.3 \\
TOTAL & 475 \\
\hline
\end{tabular}

Tabla 3. Frecuencia de las prestaciones recuperativas en cuanto a atendidos y atenciones.

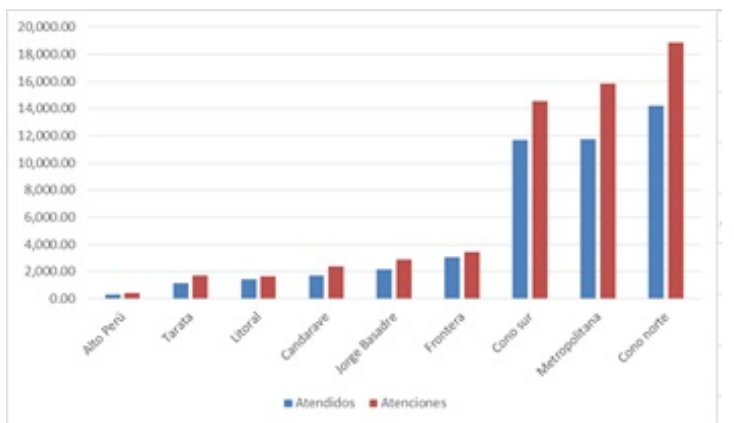

Grf. 3. Frecuencia de las prestaciones recuperativas en cuanto a atendidos $\mathrm{y}$ atenciones.

\begin{tabular}{lll}
\hline MICRO REDES & Atenciones & Ejecución Presupuestal \\
\hline Alto Perú & $\mathbf{1 , 1 5 1}$ & $\mathbf{S} / .739 .00$ \\
Candarave & 6,915 & $\mathbf{S} / .1,532.50$ \\
Cono norte & 44,451 & $\mathbf{S} / .7,716.50$ \\
Litoral & 7,636 & $\mathbf{S} / 2,274.50$ \\
Metropolitana & 44,975 & $\mathbf{S} / .8,911.50$ \\
Tarata & $\mathbf{8 , 0 3 3}$ & $\mathbf{S} / .2,985.50$ \\
Cono sur & 31,902 & $\mathbf{S} / .5,430.00$ \\
Frontera & 7,965 & $\mathbf{S} / .1,542.00$ \\
Jorge Basadre & 7,808 & $\mathbf{S} / .2,106.00$ \\
TOTAL & 160,836 & $\mathbf{S} / .33,237.50$ \\
\hline
\end{tabular}

Tabla 4. Ejecución presupuestal y cantidad de atenciones preventivas.

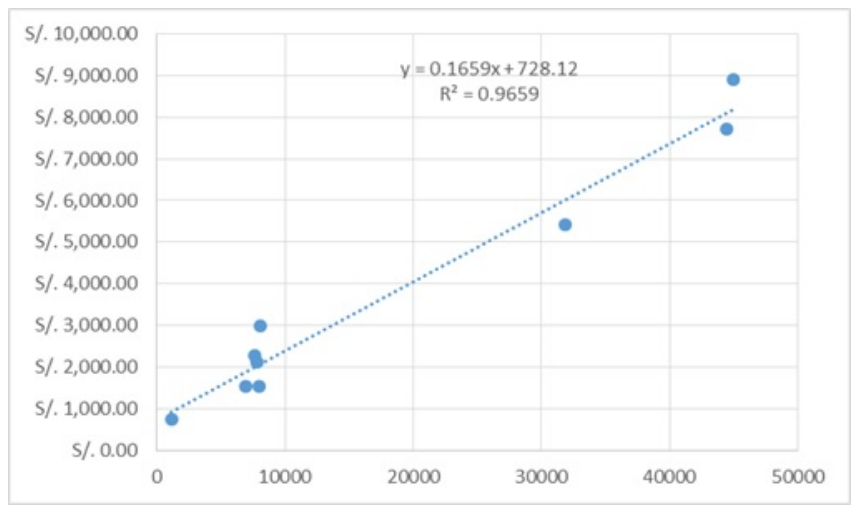

Grf. 4. Frecuencia de las prestaciones recuperativas en cuanto a atendidos y atenciones.

\begin{tabular}{|c|c|c|}
\hline MICRO REDES & Atenciones & Ejecución Presupuestal \\
\hline Alto Perú & 400 & S/.611.50 \\
\hline Candarave & 2,366 & S/. 976.00 \\
\hline Cono norte & 18,898 & S/. $12,740.50$ \\
\hline Litoral & 1,651 & S/. 2,216.50 \\
\hline Metropolitana & 15,873 & S/. $14,245.50$ \\
\hline Tarata & 1,704 & S/. 3,540.00 \\
\hline Cono sur & 14,532 & S/. $13,340.50$ \\
\hline Frontera & 3,442 & S/. 1,690.50 \\
\hline Jorge Basadre & 2,916 & S/. 3,954.50 \\
\hline TOTAL & 61,782 & S/. $53,315.50$ \\
\hline
\end{tabular}

Tabla 5. Ejecución presupuestal y cantidad de atenciones recuperativas. 


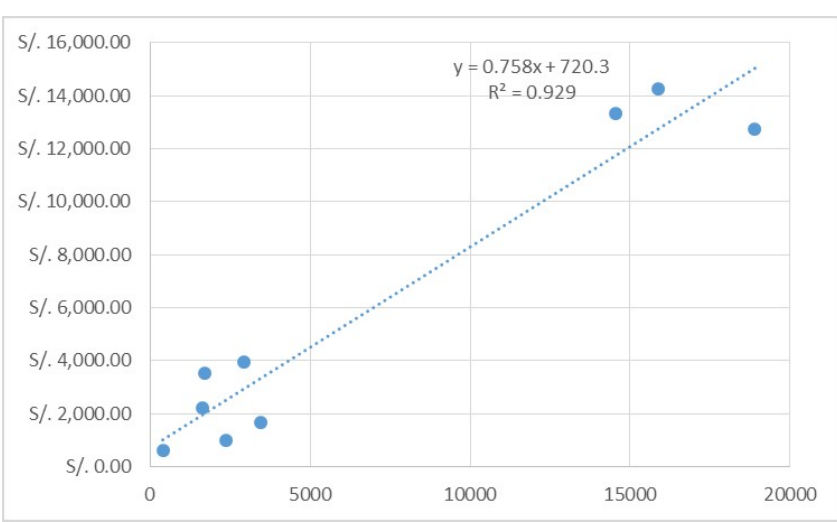

Grf. 5. Correlación entre ejecución presupuestal y cantidad de atenciones recuperativas.

afecciones bucodentales son la 2da causa de morbilidad en el analisis de la situación de Tacna, es decir hay una gran cantidad de pacientes con necesidad de tratamiento dental. La mayor concentración entre atendidos y atenciones es de 1.4 de las microredes Tarata y Alto Perú.(tabla 3 y gráfico 3). Podemos apreciar que la cantidad de presupuesto gastado está en relación con la cantidad de atenciones preventivas, sin embargo si nos detenemos a ver, esta regla no se cumple en todas debido a que hay 5 redes de salud que tienen similar cantidad de atenciones, con diferente ejecución de presupuesto (tabla 4). En el análisis general, si se cumple la relación, $\mathrm{R}^{2}=0,9$; debido a que el gran número de las redes cono Norte, Metropolitana y Cono Sur, enmascaran los valores del sector con una adecuada calidad en el gasto público.(gráfico 4). Podemos apreciar que la cantidad de presupuesto gastado está en relación con la cantidad de atenciones recuperativas $\left(\mathrm{R}^{2}=0.92\right)$, (gráfico 5) sin embargo si nos detenemos a ver el extremo inferior izquierdo esta regla no se cumple debido a que hay 5 redes de salud que tienen similar cantidad de atenciones, con diferente ejecución de presupuesto. En el análisis general, si se cumple la relación debido a que el gran n número de las redes cono norte, metropolitana y cono sur, enmascaran los valores del sector con una adecuada calidad en el gasto público.(cuadro 5)

\section{DISCUSIÓN}

El Presupuesto por Resultados (PpR) se implementa progresivamente a través de los programas presupuestales, las acciones de seguimiento del desempeño sobre la base de indicadores, las evaluaciones y los incentivos a la gestión, entre otros instrumentos que determine el Ministerio de Economía y Finanzas, a través de la Dirección General de Presupuesto Público, en colaboración con las demás entidades del Estado. En el caso de Perú, el Presupuesto por Resultados (PpR) se rige por el Capítulo IV "Presupuesto por Resultados (PpR)" en el Título III, "Normas Complementarias para la Gestión
Presupuestaria", de la Ley No 28411, Ley General del Sistema Nacional de Presupuesto. Para el Economista Samuel Torres, la adecuada asignación de recursos del Estado, se debe considerar las necesidades de la sociedad y no de individuos particulares. El Economista y docente de la Universidad del Pacifico Cristhian León nos dice que una de las maneras de lograr los objetivos que espera la población es mejorando la calidad del gasto Publico a través del Presupuesto por resultados. En nuestro país las enfermedades bucales constituyen un grave problema de Salud Pública debido a la alta prevalencia de las mismas, donde la caries dental constituye una de las primeras causas de morbilidad general, enfermedad que aqueja a más del $90 \%$ de la población nacional y a más del $95 \%$ de la Regional. nuestro país las enfermedades bucales constituyen un grave problema de Salud Pública debido a la alta prevalencia de las mismas, donde la caries dental constituye una de las primeras causas de morbilidad general. En los 10 años de experiencia profesional, he observado que la asignación presupuestal para el componente de salud bucal a tenido muchas formas de programación, siendo la más reciente, la metodología de presupuesto por resultados, donde de preconiza que el gasto público debe ser realizado, mediante el logro de metas físicas en personas atendidas. El sistema debiera garantizar que a mayor presupuesto en salud bucal, debieran haber mayor cantidad de tratamientos realizados, la hipótesis de esta investigación precisamente va a la evaluación de la veracidad de la mencionada relación.

\section{CONCLUSIONES}

Primera.- En el año 2012 se asignó un presupuesto total de S/.245,580.00 nuevos soles de los cuales se ejecutaron S/. $86,553.00$ nuevos soles.

Segunda.- El porcentaje de ejecución de tratamientos preventivos fue del $80 \%$; en tratamientos recuperativos fue de $111 \%$ ( se ejecutó más de lo programado) y en tratamientos especializados fue de $0 \%$.

Tercera.- Existe correlación entre el presupuesto asignado / ejecutado con la cantidad de tratamientos dentales, debido a que hay correlación estadísticamente significativa. Sin embargo esta relación no se cumple en el $100 \%$ de micro redes. Cuarta.- La inversión en prevención es más rentable que la inversión en Rehabilitación.

\section{Referencias}

1 Alvarado, Betty y otros. Perú hacia un presupuesto por resultados: Afianzando la transparencia y rendición de cuentas. Publicado por Universidad del Pacifico: Perú; 2011.

2 Armijo, Marianela. Diagnóstico Preliminar de la Gestión por Resultados en el Perú: 2009.

3 Barrantes, Roxana.. Los Fondos Públicos o de cómo el Presupuesto Público va perdiendo capacidad redistributiva. Documento de Trabajo $N^{\circ} 152$ del Consorcio de Investigación Económica y Social: Perú ;2009. 
4 Derechos de Participación Ciudadana en la Legislación Peruana en el ámbito de los Gobiernos Locales Fovida: Peru ;2009.

5 Flores Soria, J.. Contabilidad Gerencial. 1a ed. Peru; 2010.

6 Guzmán, Marcela. Sistema de Control de Gestión y Presupuesto por Resultados: Chile; 2012.

7 Manual de Definiciones Operacionales del PPR MINSA 2011.

8 Marcel Mario. Presupuesto por Resultado, Aspectos conceptuales y experiencias internacionales. En Taller Nacional de Efectividad en el Desarrollo para Ejecutivos de Alto Nivel. Ministerio de Economía y Finanzas: Perú; 2011

9 Ministerio de Economía y Finanzas. Los programas estratégicos. Articulado nutricional .1a ed. Perú; 2001

10 Rojas, F. Presupuesto por resultado. Diseño de programas de una guía práctica. Publicado por centro superior de estudios de administración y finanzas públicas para el desarrollo: Paraguay; 2013.

11 Torres Palacios, Valentín. Presupuesto basado por resultados. Publicado por la Secretaria de hacienda y de crédito publico: México; 2009.

Recibido: 20 de Enero de 2016

Aceptado: 27 de Enero de 2016 